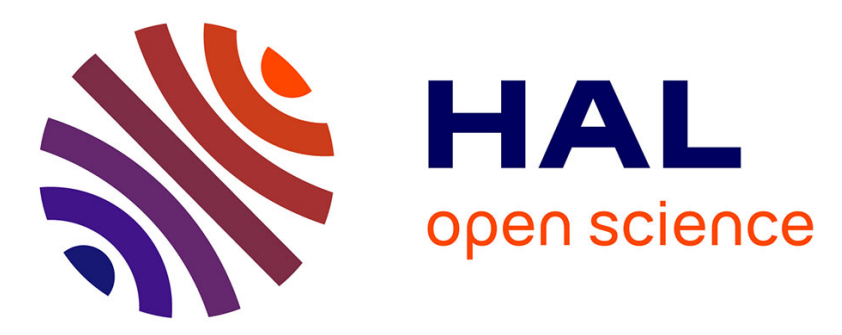

\title{
Take-over control paradigms in collaborative virtual environments for training
}

Gwendal Le Moulec, Ferran Argelaguet Sanz, Anatole Lécuyer, Valérie Gouranton

\section{- To cite this version:}

Gwendal Le Moulec, Ferran Argelaguet Sanz, Anatole Lécuyer, Valérie Gouranton. Take-over control paradigms in collaborative virtual environments for training. ACM Symposium on Virtual Reality Software and Technology (VRST), 2016, Munich, Germany. hal-01393003

\section{HAL Id: hal-01393003 https://hal.science/hal-01393003}

Submitted on 5 Nov 2016

HAL is a multi-disciplinary open access archive for the deposit and dissemination of scientific research documents, whether they are published or not. The documents may come from teaching and research institutions in France or abroad, or from public or private research centers.
L'archive ouverte pluridisciplinaire HAL, est destinée au dépôt et à la diffusion de documents scientifiques de niveau recherche, publiés ou non, émanant des établissements d'enseignement et de recherche français ou étrangers, des laboratoires publics ou privés. 


\section{Take-Over Control Paradigms in Collaborative Virtual Environments for Training}

\author{
Gwendal Le Moulec* \\ INSA de Rennes, IRISA, Inria, France
}

\author{
Ferran Argelaguet ${ }^{\dagger}$ \\ Inria, France
}

\author{
Anatole Lécuyer ${ }^{*}$ \\ Inria, France
}

\author{
Valérie Gouranton ${ }^{\S}$ \\ INSA de Rennes, IRISA, Inria, France
}

\begin{abstract}
The main objective of this paper is to study and formalize the TakeOver Control in Collaborative Virtual Environments for Training (CVET). The Take-Over Control represents the transfer (the take over) of the interaction control of an object between two or more users. This paradigm is particularly useful for training scenarios, in which the interaction control could be continuously exchanged between the trainee and the trainer, e.g. the latter guiding and correcting the trainee's actions. The paper presents the formalization of the Take-Over Control followed by an illustration focusing in a use-case of collaborative maritime navigation. In the presented use-case, the trainee has to avoid an under-water obstacle with the help of a trainer who has additional information about the obstacle. The use-case allows to highlight the different elements a Take-Over Control situation should enforce, such as user's awareness. Different Take-Over Control techniques were provided and evaluated focusing on the transfer exchange mechanism and the visual feedback. The results show that participants preferred the Take-Over Control technique which maximized the user awareness.
\end{abstract}

Index Terms: I.3.6 [Computer graphics]: Methodology and Techniques-Interaction techniques; I.3.7 [Computer graphics]: Three-Dimensional Graphics and Realism-Virtual reality

\section{INTRODUCTION}

Collaborative Virtual Environments for Training aim at training one or several trainees to perform specific tasks, which are potentially collaborative [4]. In general, a trainer is present and can interact with the virtual environment in order to assist and guide the trainees to succeed in their tasks [6]. In some cases, the trainer needs to interrupt a trainee who is acting wrong, and take control of the interaction in order to show the right way to do it. The typical use-case is a driving lesson: the driving instructor can gain control of the car in case of need. In such situation, the control of the interaction is transferred from the trainee to the trainer. This process will be called "Take-Over Control" in the remainder of the paper. Although previous works have explored related concepts like co-manipulation and live assistance to the trainee (e.g step by step guiding in order to help a trainee to perform a task [2]), the TakeOver Control has not been formally studied yet in Virtual Reality.

The main contributions of this paper are the formalization of the Take-Over Control by detailing the main elements of the CVET involved and their relationships and an illustration of the proposed formalism through a use-case of maritime navigation, where a trainee has to avoid an under-water obstacle with the help of a trainer. A user-study is then presented. Its purpose is to evaluate the trainee's awareness regarding the Take-Over Control. The training

\footnotetext{
*e-mail: gwendal.le-moulec@irisa.fr

†e-mail: fernando.argelaguet_sanz@inria.fr

‡e-mail: anatole.lecuyer@inria.fr

§e-mail: valerie.gouranton@irisa.fr
}

effects are not studied. The remainder of this paper is organized as follows: first we present a state of the art in Section 2. Then we present our formalism in Section 3. At last, we present the application of the formalism on an application of maritime navigation along with the pilot user study in Section 4.

\section{State of the ARt}

Although the notion of Take-Over Control has not been specifically addressed yet, a number of previous works have proposed interaction paradigms sharing common elements with the Take-Over Control. Control exchange has been studied in avatars, Lopez et al. [5] experimented exchanges of avatars between actors of the same CVET. A user triggering an exchange gains control over the avatar of another user. It is not exactly a Take-Over Control situation, since all control of the former avatar is lost. However the two concepts are close. The authors compared several metaphors in order to provide a feedback to the users. One of them consists in making appear phantom-like replicas of two avatars about to be exchanged. The phantoms translate to their new avatar. In addition, color codes were also used in order to identify each user. Still, these metaphors are specific to avatar case.

Visual feedback design for collaborative manipulation also benefits from color codes: Aguerreche et al. [1] designed a metaphor where users are bound to an object they manipulate collaboratively. The bounds are represented by pointers of different colors, each color being assigned to a user. The influence of a user on the control of an object depends on the distance between the user and the object. In this scenario, the level of control for each user is represented by a virtual rubber-band which changes its color according to the distance between the user and the object. Another example is the Bent Pick Ray technique which allows multiple users to collaboratively manipulate virtual objects from the distance using virtual rays [7]. The virtual rays are deformed according to the input of both users increasing the user awareness. Explicit representations of a user contribution to the interaction has been also studied. García et al. [3] proposed a visual feedback consisting of a vector showing where an actor is trying to move a virtual beam.

Collaborative manipulation was also studied as a mean to help a user to perform a task. Pinho et al. [6] studied the case of asymmetric manipulation. Two actors have to insert an object in a small, reserved place. The main actor controls only the translations while the assistant actor controls the rotations. This work division makes the task easier. Ruddle et al. [9] studied other ways to integrate the contributions of two different users of the same collaborative manipulation interaction. The resulting movement can be either the sum, the mean or the intersection (keeping of the common parts) of the contributions.

\section{Theoretical Framework and Formalism}

This section presents the formal definitions of the shared interaction control concept (Section 3.1) and of the Take-Over Control (Section 3.2). Two subsequent components of the Take-Over Control are then characterized: weight functions and user awareness. 


\subsection{Shared interaction control}

Let $a$ be an actor and $o$ an object. Then, " $a$ controls $o$ " means that " $a$ can interact with $o$ ". Therefore "performing a Take-Over Control on an object" means "gaining ability to use interactions related to an object, to the detriment of another actor". Consequently, the central concept is "ability to use an interaction". In the collaborative case and in the remainder of the paper it will be called "shared interaction control". A shared interaction control is composed of three elements: a set of actors, an object and an interaction on this object (see Figure 1).

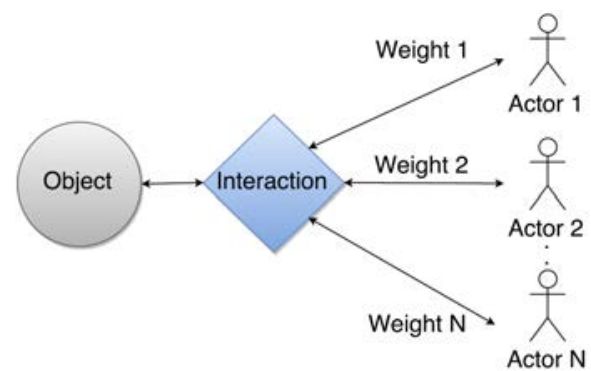

Figure 1: Shared interaction control with $N$ actors. Control of the interaction is balanced by weights.

Definition: let $s c_{o}$ be a shared interaction control on an object $o$. Then the definition of $s c_{o}$ is given by the set membership relation of Equation 1, where $A$ is the actor set of the CVET, $I_{O}$ the set of available interactions with $o$ and $M$ a number of $\mathbb{R}^{*+}$.

$$
s c_{o} \in\left(I_{o} \times(A \rightarrow[0, M])\right)
$$

The positive real numbers associated to each actor are weights determining how the contribution of an actor to the interaction is taken into account. A weight equal to 0 means that the actor does not control the object at all. $M$ is an upper-bound let free in the definition. For clarity concerns, $M=1$ in the remainder of the paper.

Note that the case of non-collaborative interactions is a particular case of shared interaction control. Consider a non-collaborative interaction on an object in a CVET. Such an interaction is characterized by a constraint on weights, expressed by Equation 2, where each $w_{i}$ is the weight associated to the actor identified by $i$, each actor being identified by a number of $\{1, \ldots, n\}$, the quantity $n$ being the number of actors in the considered CVET.

$$
\exists ! i \in\{1, \ldots, n\}: \forall j \in\{1, \ldots, n\} \begin{cases}w_{j}=1, & \text { if } j=i \\ w_{j}=0, & \text { otherwise }\end{cases}
$$

\subsection{Take-Over Control}

The definition of the Take-Over Control consists essentially in modeling the process of gaining/losing the ability to use an interaction.

Definition: Consider a CVET with $n$ actors, a positive real number $T$ and the function over time $T O C_{o}:[0, T] \rightarrow S C_{o}$ such that for each $t \in[0, T], T O C_{o}(t)$ is a shared interaction control of parameters $\left(i_{o}, f_{n}(t)\right)$, where $f_{n}(t)=\left\{\left(a_{1}, w_{1}(t)\right), \ldots,\left(a_{n}, w_{n}(t)\right)\right\}$, each $a_{i}$ being the actor $i$ and each $w_{i}$ being the associated weight function (over time). Then, $T_{O C}$ is a Take-Over Control if and only if it respects the constraint defined by Equation 3 .

$\exists i, j \in\{1, \ldots, n\}, i \neq j: w_{i}(0)=0, w_{j}(0)=1, w_{i}(T)=1, w_{j}(T)=0$

Equation 3 means that $a_{i}$ is the actor who gains control and that $a_{j}$ is the actor who loses it.

In real use-cases, choosing a weight function rather an other is likely to have consequences on the user-experience. For instance, a sudden loss of control could generate some discomfort for the trainee. A sudden control gain is here characterized by functions of the form given by Equation 4, $a$ being a real number of $[0, T]$.

$$
w(t)= \begin{cases}0, & \text { if } t \in[0, a] \\ 1, & \text { if } t \in] a, T]\end{cases}
$$

A smooth control gain would be characterized by a weight function with intermediate values and a value of $T \geq 1 \mathrm{~s}, 1$ second being the average reaction time. An example is the function of Equation 5.

$$
w(t)=\frac{t}{T}
$$

The definitions along with Figure 1 show that in order to provide a good Take-Over Control awareness, weight functions must be clearly represented, as well as the weight-actor associations. The interaction must be represented too and more importantly the effect resulting from the combination of all the contributions (weighted by each actor's weight). In other words, the feedback must ensure the awareness of the object, the interaction, the weight functions, the different contributions and the resulting action.

\section{Use-CASE: Maritime NaVigation}

The following use-case is a training application based on a concern of real-life navigation: under-water obstacles avoidance. Underwater obstacles can be rocks or coral for example. They are not visible and that is why a novice boat driver must be accompanied by an expert. The virtual environment consists of a boat floating on a calm sea. There are two users: a trainee and a trainer. The boat is controlled by the trainee, who has to avoid the under-water obstacles. The trainer can perform a Take-Over Control if the trainee is about to collide with the obstacle (Figure 2).

\subsection{Take-Over Control characterization}

In this use-case, the Take-Over Control is characterized by the helm of the boat (object $o$ ) and the actor set $A=\left\{a_{1}, a_{2}\right\}$, with $a_{1}$ and $a_{2}$ being the trainee and the trainer respectively. The weight functions are $w_{1}$ and $w_{2}$ for the trainee and the trainer respectively. They respect the constraint expressed by Equation 7. The interaction set $I_{O}$ (singleton) is the control of the boat deviation $\theta$, expressed by Equation 6 in function of $\theta_{1}(t)$ and $\theta_{2}(t)$, respectively the deviation requested by the trainee and the deviation requested by the trainer at the instant $t$.

$$
\begin{gathered}
\theta(t)=w_{1}(t) \times \theta_{1}(t)+w_{2}(t) \times \theta_{2}(t) \\
w_{1}(t)=1-w_{2}(t)
\end{gathered}
$$

\subsection{Take-Over Control visual feedback}

In this section, the design of the Take-Over Control visual feedback is presented. This feedback is illustrated in Figure 2. In order to improve awareness, it takes into account all the elements of the formalism: the object, the interaction, the actors' contributions and the weight functions.

Representation of the object and of the interaction. The shared object $(o)$ is the helm (see Figure 2). The interaction consists in making the helm rotate. The angle of rotation $(\theta)$ is represented thanks to a reference point on the helm: a white handle. Its deviation from its initial vertical position (see Figure $2 b$ ) gives the angle value. For example, in Figure $2 \mathrm{c}, \theta \approx-45^{\circ}$.

Representation of the actors' contribution. The solution is inspired from the phantom-like replicas [5]. The helm is given a symbolic replica, placed before it: a disk with an arrow. The goal of this replica is to represent the contribution of the trainer $\left(\theta_{2}\right)$. As for the white handle, the deviation of the arrow from its initial vertical position (see Figure $2 b$ ) gives the value of $\theta_{2}$. For example, 


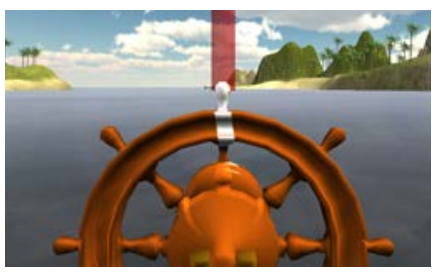

(a) Initial state

No feedback condition

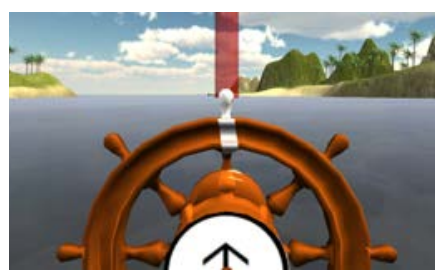

(b) Initial state

$w_{1}=1, w_{2}=0$

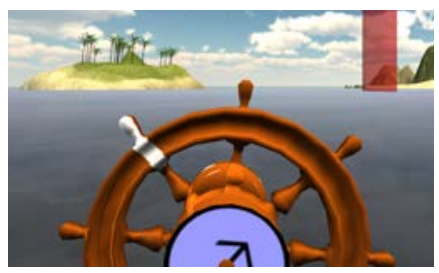

(c) In progress

$w_{1}=0.5, w_{2}=0.5$

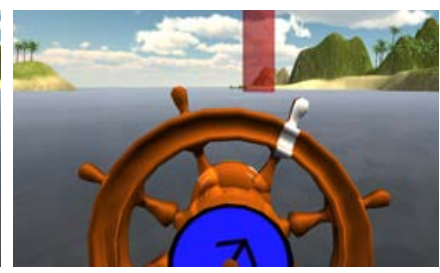

(d) Completed

$w_{1}=0, w_{2}=1$

Figure 2: Illustrations depicting the two visual feedback conditions considered in the use case. The user was instructed to steer the boat towards the semitransparent red column (target destination) by controlling the heading of the boat. The white handle indicated the rotation angle of the boat. Figure (a) shows the feedback for the no-feedback condition. Sequence (b,c,d) shows the evolution of the contribution of the trainer on the steering angle, from no control to total control.

in Figure $2 \mathrm{c}, \theta_{2} \approx 45^{\circ}$. The contribution of the trainee is directly represented by the used device (e.g a joystick).

Representation of the weight functions. The disk is initially white (Figure $2 b$ ). It becomes blue when the trainer controls the boat (Figures $2 \mathrm{c}$ and $2 \mathrm{~d}$ ). The opacity of the blue corresponds to the trainer weight value (transparent if $w_{2}=0$, opaque if $w_{2}=1$ ). Representing the trainee weight function is not necessary because of the constraint on the weight functions (Equation 7).

\section{USER Study}

The objective of the user study was to asses the preferences of the users regarding two aspects of the proposed approach: (1) presence or absence of feedback regarding trainer activity and (2) the scheme of the Take-Over Control, i.e sudden vs. smooth. The two conditions of visual feedback and the two control laws were evaluated.

Participants played the role of the trainee. The trainer was a virtual user, i.e a simple algorithm making the boat trajectory deviate in order to avoid the obstacle. The Take-Over Control is indeed studied from the trainee point of view only; a virtual trainer ensures that all the participants are evaluated under the same conditions. The trainees had to steer a virtual boat towards a specified location in the virtual environment. At first glance, the target location could be reached following a straight path. However there was an under-water (invisible) obstacle placed at the middle of this path.

The participants were told about this and were asked to try to reach the target without worrying about the obstacle, until the intervention of the trainer in order to avoid it, thanks to the Take-Over Control. They had then to follow as best as they could the trajectory they thought the trainer was taking. When the obstacle was passed, the trainer released the control and the participants had to follow the target again. The obstacle was not displayed in purpose, as it required the virtual trainer intervention in order to successfully avoid it. The quality of the avoidance task had no importance in itself. There was no notion of "best path". The goal was not to evaluate training performances either. The focus was on user awareness.

\subsection{Experimental protocol and design}

Eight participants took part in the pilot study (age $M=27.1 S D=6.1$ ). The experiment was conducted using a desktop computer and participants interacted with the virtual environment using a joystick.

Upon arrival, participants were asked to read and sign the experiment consent form that provided written instructions about the experiment. At the end of the experiment participants were asked to answer a subjective questionnaire.

The experiment had three within-subject factors, the visual feedback (present/absent), the weight functions type (sudden/smooth, of the form given by Equations 4 and 5 respectively) and the size of the obstacle $(20 \mathrm{~m} / 40 \mathrm{~m})$. To minimize ordering effects, the order of the visual feedback and the weight functions type were counterbalanced. The size of the obstacle was randomized. For each combination, participants performed 6 repetitions, resulting in a total of $2 \times 2 \times 2 \times 6=48$ trials. In addition, at the beginning of the experiment, participants performed several training trials to understand the task and the navigation control. Although the trajectories were recorded for each trial, the main purpose of the experiment was to gather subjective data from participants. To do so, at the end of the experiment, participants were asked to subjectively rate the visual feedback (usefulness, information transfer, appreciation, awareness) and the weight functions type (pleasantness, easiness, encumbrance, appreciation) on a 7-point Likert scale. The main hypotheses were that participants would prefer the smooth control over the sudden control [H1], and an increased feeling of awareness was expected when the visual feedback was present [H2].

\subsection{Results}

The data gathered from the questionnaires were analyzed using the non-parametric Wilcoxon pairwise test. A summary for the questionnaire results can be observed in Figure 3. The subjective ratings for the weight function types showed a significant effect for the level of appreciation $(p<0.05)$ and pleasantness $(p<0.05)$. Participants in overall, preferred the smooth transition over the sudden one. In contrast, no significant differences were found for the encumbrance $(p=0.106)$ nor the easiness $(p=0.107)$. These results support [H1], however, in terms of interaction both methods are perceived similarly. Regarding the subjective ratings of the visual feedback, a significant increase of the level of awareness $(p<0.05)$ and information transfer $(p<0.05)$, and an almost significant increase for the level of appreciation $(p=0.052)$ could be observed when the visual feedback was present. In contrast, no significant increase for the level of usefulness $(p=0.178)$ was observed. These results also supports [H2] as the addition of the visual feedback has a positive effect regarding awareness and information transfer.

\subsection{Discussion}

The visual feedback appears to be significantly appreciated, and provides a better user awareness. Even if this result could be strongly guessed, it should be remembered that without the visual feedback, the movements of the helm bring some information about the contribution of the trainer. It is then interesting to note that visually separating the trainer contribution from the resulting action has its importance.

The study also shows that there are no significant differences in terms of felt usefulness when there is a visual feedback and when there is not. That means that the participants do not feel that the metaphor brings a significant help for performing the task.

It was assumed that smooth weight functions would avoid some discomfort. The experiment has shown that indeed the smooth type 

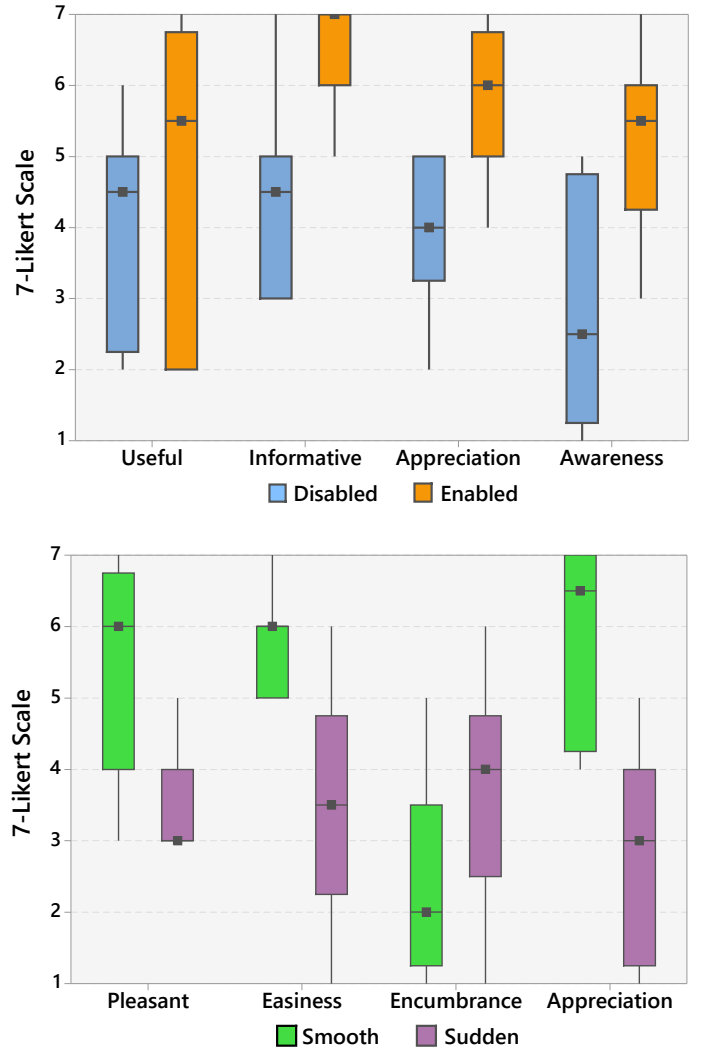

Figure 3: Boxplots for the subjective questionnaires. First, subjective ratings for the different levels of visual feedback. Second, subjective ratings for the different types of weight functions.

was preferred on this example, but this conclusion can not be generalized. It would be necessary to compare several smooth and sudden functions on different use-cases, adapted to the Take-Over Control and known in the literature $[8,3]$.

\section{Conclusion}

In this paper, the concept of Take-Over Control has been formally introduced and defined as a shared interaction control evolving over time such that the control is transferred between several users. The contributions for each user are balanced using weight functions (e.g. smooth and sudden) which allow to change the nature of the Take-Over Control. The proposed framework was then applied to an application of maritime navigation in CVET focusing on the main components of the Take-Over Control metaphor: the interactive objects, the interactions, the actor's contributions and the weight functions. The evaluation of the different proposed solutions showed that user's tend to appreciate increased user awareness both in terms of visual feedback and control (smooth weight function). This use-case showed how the proposed framework can be used to design and evaluate collaborative interactions.

For future works, the formalism should be tested on other usecases. Studying the influence of the several techniques in different contexts is indeed necessary to complete the Take-Over Control design. In particular, these studies would allow to evaluate the sudden weight functions in situations where reactivity is important. Notably, a particular situation should be studied: performing a TakeOver Control on the trainee avatar rather than on a simple object. It would raise more "social" concerns ; the generated discomfort is of different nature. The formalism could be enriched in order to take this case into account.

\section{REFERENCES}

[1] L. Aguerreche, T. Duval, and A. Lécuyer. 3-hand manipulation of virtual objects. In $E G V E$, pages 153-156, 2009.

[2] T. Duval, T. T. H. Nguyen, C. Fleury, A. Chauffaut, G. Dumont, and V. Gouranton. Improving awareness for $3 \mathrm{~d}$ virtual collaboration by embedding the features of users physical environments and by augmenting interaction tools with cognitive feedback cues. Journal on Multimodal User Interfaces, 8(2):187-197, 2014.

[3] A. S. García, J. P. Molina, D. Martínez, and P. González. Enhancing collaborative manipulation through the use of feedback and awareness in cves. In VRCAI, pages 45-50, 2008.

[4] M. Hirose, D. Schmalstieg, C. Wingrave, and K. Nishimura. Collaborative interaction in co-located two-user scenarios. EGVE - ICAT, pages $85-92,2009$.

[5] T. Lopez, R. Bouville, E. Loup-Escande, F. Nouviale, V. Gouranton, and B. Arnaldi. Exchange of avatars: Toward a better perception and understanding. IEEE TVCG, 20(4):644-653, 2014

[6] M. S. Pinho, D. A. Bowman, and C. M. Freitas. Cooperative object manipulation in immersive virtual environments: Framework and techniques. In ACM VRST, pages 171-178, 2002.

[7] K. Riege, T. Holtkämper, G. Wesche, and B. Fröhlich. The bent pick ray: An extended pointing technique for multi-user interaction. In IEEE $3 D U I$, pages 62-65, 2006.

[8] D. Roberts, R. Wolff, O. Otto, and A. Steed. Constructing a gazebo: supporting teamwork in a tightly coupled, distributed task in virtual reality. Presence, 12(6):644-657, 2003.

[9] R. A. Ruddle, J. C. Savage, and D. M. Jones. Symmetric and asymmetric action integration during cooperative object manipulation in virtual environments. ACM TOCHI, 9(4):285-308, 2002. 